\title{
1 Detection of Reticuloendotheliosis Virus in Brazil
}

3 Giovana S. Caleiro ${ }^{1}$, Cristina F. Nunes ${ }^{1}$, Paulo R. Urbano ${ }^{1}$, Karin Kirchgatter ${ }^{2}$, Jansen de 4 Araujo $^{3}$, Edison Luiz Durigon ${ }^{3}$, Luciano M. Thomazelli ${ }^{3}$, Brittany M. Stewart ${ }^{4}$, Dustin C. 5 Edwards ${ }^{4}$, and Camila M. Romano ${ }^{1}$

$7 \quad{ }^{1}$ Instituto de Medicina Tropical de São Paulo e Hospital das Clínicas da Faculdade de Medicina 8 -HCFMUSP (LIM52) Av Dr. Enéas Carvalho de Aguiar, 470 - CEP 05403-000 -São Paulo/SP 9 Brasil, Universidade de São Paulo

${ }^{2}$ Instituto de Medicina Tropical de São Paulo, SUCEN, Av Dr. Enéas Carvalho de Aguiar, 470 CEP 05403-000 - São Paulo/SP - Brasil

3 Laboratório de Virologia Clínica e Molecular, Instituto de Ciências Biomédicas (ICB), Universidade de Sao Paulo, Av. Prof. Lineu Prestes, no 2415CEP 05508-900 - Butantã - São Paulo/SP, Brasil

${ }^{4}$ Tarleton State University, Department of Biological Sciences, Box T-0100, Stephenville, Texas $19 \quad 76402$

20

21 Corresponding Author: Camila M. Romano Av Dr. Enéas Carvalho de Aguiar, 470-CEP 05403-

22 000, São Paulo/SP, BrasilPhone: 55113061-8668

23 email: cmromano@usp.br 


\section{Abstract}

31 Reticuloendotheliosis retroviruses (REV) are known to cause immunosuppressive and oncogenic

32 disease that affects numerous avian species. REV is present worldwide and recently has been

33 reported in South America with cases of infected commercial flocks in Argentina. We surveyed

34 for the presence of REV in birds from a state in the northern region of Brazil using real-time

35 PCR. We report the first cases of REV in Brazil, detected in Muscovy ducks (Cairina moschata),

36 wild turkeys (Meleagris gallopavo), and chickens (Gallus gallus) at a relatively high prevalence

37 rate $(16,8 \%)$. Phylogenetic analysis indicated a close relationship of this strain to variants in the

38 United States. This study provides evidence of REV in the Amazon biome and provides a

39 baseline for future surveillance of the virus in the region and throughout Brazil.

40

41 Key words: avian retrovirus, Brazil, reticuloendotheliosis virus 


\section{Main Text}

Reticuloendotheliosis viruses (REV) are a group of immunosuppressive and sometimes oncogenic retroviruses that affect numerous species of birds, including waterbirds

47 (Anseriformes), game birds (Galliformes), and perching birds (Passeriformes) (Nair et al. 2013). REV has previously affected commercial poultry and has been a recurring obstacle in the

49 conservation of endangered species (Luan et al. 2016). Within vaccinated flocks, REV infection can lead to decreased efficacy of vaccinations for avian influenza virus, Newcastle disease virus,

51 Marek's Disease virus (MDV), and turkey herpesvirus due to the reduced humoral response 52 resulting from immunosuppression (Sun et al. 2009). The virus was first identified in the US in

53 1958, and later in China, Taiwan, Australia, Argentina, and Canada (Singh et al. 2003;

54 MacDonald et al. 2018). REV prevalence varies from 0-50\% depending on the region, setting, 55 and avian species (Jiang et al. 2013; Ferro et al. 2017).

Evidence of REV in South America was recently demonstrated in fowlpox-vaccinated commercial poultry flocks in Argentina (Buscaglia, 2013). REV proviral DNA has been detected in the genomes of the attenuated MDV vaccine (MD-2 strain) and the field and vaccine strains of

59 fowlpox viruses (FWPV-REV) (Isfort et al. 1992; Hertig et al. 1997). Vaccine-integrated REV can be either infectious or non-infectious (Hertig et al. 1997; Moore et al. 2000). The Brazilian

61 coast serves as an important stopover site for migratory birds coming from the northern

62 hemisphere. Several species migrate during the austral winter from Argentina and Chile to

63 Central Bolívia and Brazil (Somenzari et al. 2018). In addition, several birds, including 64 passeriformes, migrate from the US, Mexico, and Central America to northern Brazil (Somenzari 65 et al. 2018). However, no prior studies have surveyed for the presence of REV in Brazil. With 66 more than 2,000 species of wild birds present in the country, many of which are endangered, 
67 determining the presence of REV and establishing a baseline prevalence rate could be of value

68 for future conservation efforts in the Amazon biome.

During 2005-2006, a total of 441 samples were collected (blood and pooled

70 cloacal/orotrachea swabs) near eight different cities in the northeastern region of Pará state. Most

71 of the samples were from Muscovy ducks (Cairina moschata, $n=379$ ), and the remaining

72 samples were from turkeys (Meleagris gallopavo, $n=41$ ), and chickens (Gallus gallus, $n=21$ ).

73 Total cDNA from orotrachea and cloacal swab samples was obtained and previously prepared

74 from an avian influenza virus detection study (Thomazelli et al. 2012). As an internal control, a

75 conventional PCR method for avian mitochondrial DNA was performed using cytochrome $b$

76 primers (Kocher et al. 1989). The presence of REV proviral cDNA was detected using real-time

$77 \quad$ PCR and primers specific to the $g p 90$ gene (env) (Li et al. 2013). Reactions were prepared using

$782.5 \mathrm{mM}$ of each primer, $7.5 \mu \mathrm{L}$ of water, $12.5 \mu \mathrm{L}$ of MasterMix SYBR ${ }^{\circledR}$ Green

79 (LifeTechnologies ${ }^{\circledR}$, Brazil), and $3 \mu \mathrm{L}$ of purified cDNA. Plasmids containing genes of interest

80 were constructed from commercially-synthesized inserts (GenScript, USA) to serve as positive

81 controls. In addition, partial fragments of LTR-U5, Gag, and envelope from REV-positive

82 samples were amplified and sequenced by Sanger method using previously described primers

83 (Singh et al. 2003; Barbosa et al. 2007; Li et al. 2013). For the envelope region, an additional

84 primer was designed (gp90-7242R 5'-GCCAGTATGCACAGCCCTATCCA-3’).

Of the 441 samples tested and amplified, REV PCR products were detected in 74 samples

86 (16,7\%). Infected individuals included 65 Muscovy ducks, 6 wild turkeys, and 2 chickens. REV

87 positive samples came from Marabitana, Vila Maracajá, and Marajó. Sequencing reads were

88 inspected for quality and consensus sequences were built using CLC genomic workbench v5

89 (https://www.qiagenbioinformatics.com/) and submitted to GenBank, accession numbers 
MG953804-MG953809. Consensus sequences of partial LTR/gag (808 bp), gag (1168 bp), and

91 envelope ( $\sim 600 \mathrm{bp})$ were aligned and compared to reference strains and other sequences

92 retrieved from GenBank representing worldwide REV ( $n=16)$. Phylogenetic trees were

93 reconstructed under maximum likelihood method in PhyML software using K80 as the best

94 nucleotide substitution model for all fragments as determined by jModeltest (Guindon et al.

95 2010; Darriba et al. 2012). Only sequences built with high quality reads (phred $>30$ ) were used

96 for phylogenetic reconstructions. Genetic distance ( $p$-distance) between the main REV strains

97 and one representative Brazilian REV sample was estimated for LTR/gag, gag, and envelope

98 partial fragments. To establish the phylogenetic relationships among Brazilian and worldwide

$99 \mathrm{REV}$, phylogenetic trees were reconstructed using LTR/gag and envelope fragments representing

100 Brazilian and worldwide REV genomic sequences ( $n=16)$ (Fig 1). Brazilian REV clustered

101 together with sequences sampled in the US, such as APC-566. Genetic distance at the nucleotide

102 level ( $p$-distance) between the main REV strains and one representative Brazilian REV also

103 agreed that Brazilian REV is more closely related to the US samples representative of subtype 3,

104 such as MD-2 and APC-566, rather than to spleen necrosis virus (SNV) strain or China isolates

105 (Fig. 1 and Table 1).

106

Pará state is located in the northern coastal region of Brazil and has a tropical rainforest

107 climate. Due to its geographic location and ecosystem, Pará is a stopover site for shorebirds

108 coming from the northern hemisphere during the migration season. In addition, several species of

109 migratory birds that have Argentina and the US as their final destination pass through Brazil

110 during their flight. The similarity between Brazilian REV and the US viruses suggests that the

111 US could be the source of REV detected in Brazil. However, there is no sequence data available

112 for viruses found in Argentina. As such, the source of Brazilian REV cannot be definitively 
113 determined. Representative strains of REV include the defective REV-T, the non-defective REV-

114 A, SNV, duck infectious anemia, and chicken syncytial virus (CSV), and the MD-2 and FWPV

115 vaccine-integrated strains. As there is relatively little genetic variation among REV strains, it is

116 difficult to determine the origin and classification of an isolate (Bohls et al. 2006). In our

117 analysis, Brazilian REV differs only 0.0-0.5\% from both FWPV and MD-2-integrated REVs and

118 non-integrated APC-566 and CSV. We inspected for point mutations, which have been

119 characterized as specific for FWPV-REV (Tadese et al. 2008). According to the genetic pattern

120 observed, the Brazilian REV samples are all non-FWPV-integrated strains. However, not all

121 FWPV-REV envelope sequences available on GenBank (KY498002, AF246698, and

122 JX217830) contain this signature, indicating that some genetic variability among integrated

123 strains exists and determining the integration status based on few point mutations is inaccurate

124 (Tadese et al. 2008). Therefore, it remains undetermined if Brazilian REV is present as infectious

125 particles or is instead integrated within a large DNA virus genome.

Reticuloendotheliosis viruses can infect a number of species, including captive and wild

127 perching birds, game birds, and waterbirds. Although we detected REV in Muscovy ducks, wild

128 turkeys, and chickens, we could only amplify and sequence the virus from ducks. It is possible

129 that the virus was present in a low viral load in other species, and we could not generate large

130 amplicons. Muscovy ducks are native to Mexico, Central, and South America, but populations of

131 Muscovy ducks reside in the US, mainly in Florida and southern Texas. These birds are

132 essentially non-migratory or irregular migrants without any established migration patterns, only

133 migrating short distances to avoid dry weather and fluctuating water conditions. Chickens and

134 turkeys, frequently associated with REV infections, are also non-migratory birds. Therefore, we

135 consider it is unlikely that the positive birds found in Brazil were infected elsewhere. 
Here, we detected REV in Brazil for the first time and presented the first DNA sequences of REV provirus from South America. REV found in Brazil is similar to other common

138 circulating strains, including those found in the US. Additionally, considering that REV was 139 present in samples from three of the five regions collected from Pará from 2005-2006 (located $140 \quad 200-500 \mathrm{~km}$ apart from each other), it is very likely that the virus has spread to other states in

141 Brazil. We hypothesize that the virus could be carried by migratory birds which stopover in the 142 northern part of the country on their way to and from North America. These results suggest the 143 need for additional studies to further determine the prevalence and genetic variability of REV in 144 Brazilian wild and captive birds and also to perform risk assessment studies dedicated to free145 range and captive commercial avian species.

146 All procedures involving wild and captive birds were approved by the Animal Ethics 147 Committee from the Instituto de Medicina Tropical de São Paulo under protocol 000283A, and 148 licensed by the Ministério do Meio Ambiente-MMA at the Instituto Chico Mendes de 149 Conservacão da Biodiversidade (ICMBio/SISBIO) under protocols 34605-7 45527-2, 54616-1, 150 and 201/2006 CGFAU. This work was supported by Fundação de Amparo a Pesquisa do Estado 151 de São Paulo (FAPESP Project \#2015/05958-3). G. Caleiro holds a CAPES scholarship. 


\section{References}

Barbosa T, Zavala G, Cheng S, Villegas P. 2007. Full genome sequence and some biological properties of reticuloendotheliosis virus strain APC-566 isolated from endangered Attwater's prairie chickens. Virus Res 124:68-77.

Bohls RL, Linares JA, Gross SL, Ferro PJ, Silvy NJ, Collisson EW. 2006. Phylogenetic analyses indicate little variation among reticuloendotheliosis viruses infecting avian species, including the endangered Attwater's prairie chicken. Virus Res 119:187-194.

Darriba D, Taboada GL, Doallo R, Posada D. 2012. jModelTest 2: more models, new heuristics and parallel computing. Nat Methods 9:772.

Ferro PJ, Morrow ME, Flanagan JP, Ortego B, Chester RE, Mueller JM, Lupiani B. 2017. Wild Birds, a Source of Reticuloendotheliosis Virus Infection for the Endangered Attwater's Prairie-Chicken ( Tympanuchus cupido attwateri)? J Wildl Dis 53:586-590.

Guindon S, Dufayard J-F, Lefort V, Anisimova M, Hordijk W, Gascuel O. 2010. New algorithms and methods to estimate maximum-likelihood phylogenies: assessing the performance of PhyML 3.0. Syst Biol 59:307-321.

Hertig C, Coupar BE, Gould AR, Boyle DB. 1997. Field and vaccine strains of fowlpox virus carry integrated sequences from the avian retrovirus, reticuloendotheliosis virus. Virology 235:367-376.

Isfort R, Jones D, Kost R, Witter R, Kung HJ. 1992. Retrovirus insertion into herpesvirus in vitro and in vivo. Proc Natl Acad Sci U S A 89:991-995.

Jiang L, Qi X, Gao Y, Hua Y, Li K, Deng X, Wang Q, Zhang L, Chai H, Chen Y, et al. 2013. Molecular characterization and phylogenetic analysis of the reticuloendotheliosis virus isolated from wild birds in Northeast China. Vet Microbiol 166:68-75. 
Kocher TD, Thomas WK, Meyer A, Edwards SV, Pääbo S, Villablanca FX, Wilson AC. 1989. Dynamics of mitochondrial DNA evolution in animals: amplification and sequencing with conserved primers. Proc Natl Acad Sci U S A 86:6196-6200.

Li K, Gao L, Gao H, Qi X, Gao Y, Qin L, Wang Y, Wang X. 2013. Protection of chickens against reticuloendotheliosis virus infection by DNA vaccination. Vet Microbiol 166:59-67.

Luan H, Wang Y, Li Y, Cui Z, Chang S, Zhao P. 2016. Development of a real-time quantitative RT-PCR to detect REV contamination in live vaccine. Poult Sci 95:2023-2029.

MacDonald AM, Jardine CM, Bowman J, Susta L, Nemeth NM. 2018. Detection of lymphoproliferative disease virus in canada in a survey for viruses in ontario wild turkeys ( Meleagris gallopavo). J Wildl Dis. http://dx.doi.org/10.7589/2018-01-013.

Moore KM, Davis JR, Sato T, Yasuda A. 2000. Reticuloendotheliosis virus (REV) long terminal repeats incorporated in the genomes of commercial fowl poxvirus vaccines and pigeon poxviruses without indication of the presence of infectious REV. Avian Dis 44:827-841.

Nair V, Zavala G, Fadly AM. 2013. Reticuloendotheliosis, 13th edition. In: D. E. Swayne, J. R. Glisson, L. R. McDougald, J. V. Nolan, D. L. Suarez, and Nair V., editors. WileyBlackwell, Ames, Iowa. pp. 593-604.

Singh P, Schnitzlein WM, Tripathy DN. 2003. Reticuloendotheliosis Virus Sequences within the Genomes of Field Strains of Fowlpox Virus Display Variability. J Virol 77:5855-5862.

Somenzari M, Amaral PP do, Cueto VR, Guaraldo ADC, Jahn AE, Lima DM, Lima PC, Lugarini C, Machado CG, Martinez J, et al. 2018. An overview of migratory birds in Brazil. Pap Avulsos Zool 58:3.

Sun S, Cui Z, Wang J, Wang Z. 2009. Protective efficacy of vaccination against highly pathogenic avian influenza is dramatically suppressed by early infection of chickens with 

reticuloendotheliosis virus. Avian Pathol 38:31-34.

203 Tadese T, Fitzgerald S, Reed WM. 2008. Detection and differentiation of re-emerging fowlpox 204 virus (FWPV) strains carrying integrated reticuloendotheliosis virus (FWPV-REV) by real205 time PCR. Vet Microbiol 127:39-49.

206 Thomazelli LM, Araujo J de, Ferreira C de S, Hurtado R, Oliveira DB, Ometto T, Golono M, 207 Sanfilippo L, Demetrio C, Figueiredo ML, et al. 2012. Molecular surveillance of the 208 Newcastle disease virus in domestic and wild birds on the North Eastern Coast and Amazon 209 biome of Brazil. Fundação APINCO de Ciência e Tecnologia Avícolas. Braz J Poult Sci 210 14:01-07. 
212 Table 1

213 Genetic Identity at nucleotide level between Brazilian REV and reference strains.

\begin{tabular}{lcccc}
\hline & & & REV_2036_PA_Br \\
\hline Reference & GenBank ID & LTR/gag & Gag & Env \\
\hline APC-566 & DQ387450 & $99.8 \%$ & $100 \%$ & $99.8 \%$ \\
SNV & DQ003591 & $96 \%$ & $97.5 \%$ & $96.4 \%$ \\
MD-2 & JX912710 & $99.4 \%$ & $100 \%$ & $100 \%$ \\
FPV & AF246698 & $99.6 \%$ & $100 \%$ & $100 \%$ \\
CSV & DQ237905 & NA ${ }^{\mathrm{a}}$ & $\mathrm{NA}$ & $99.5 \%$ \\
HA9901 (China) AY842951 & $96 \%$ & $98 \%$ & $97.6 \%$ \\
& & & & \\
\hline
\end{tabular}

214

215 Distance matrix ( $p$-distance)

$216{ }^{a} \mathrm{NA}$ - sequence not available

219 Figure 1

220 Maximum likelihood Phylogenetic reconstruction showing the relationships between REV from

221 Brazil and other isolates. Brazilian sequences (in red) are from Muscovy ducks (Cairina

222 moschata). The trees were made with partial sequences from REV env (A) and LTR/gag (B)

223 available at GenBank. 
A REV_1022_BR

REV_2141_BR

REV_1092_BR

REV_1025_BR

GU222415_CSV

KF305089_REV_HA1101

KU204703_GDBL1402

REV_2036_BR

GU222418_Isol11_US

REV_USA

FJ439119_REV_goose341006

_ DQ387450_REV_APC566

KJ909531_REV_CY1111

KU641115_REV_LN1201

KU204702_GDBL1401

KJ756349_104865_turk_US

GU222417_Isol7_US

GU222416_Isol3_US

GQ415647_JSRD0701

FJ439120_chicken_3337_05

KY498002Fowlpox_Ind_Guj2011

JQ804915_REV_1105

FJ439120_REV_chicken333705

JX912710_REV_MD2

KC884561_DBYR1106

GQ415646_REV_HLJR0901China

_KC884558_DBYR1202

JX217829_Kr_ADL102544_2010

_KJ909530_REV_SY1209

KP151492_Egypt_RE_2014

KP151493_Egypt_RE_2014

100\% AY842951_REV_HA9901China

NC_006934_REV_HA9901China
B

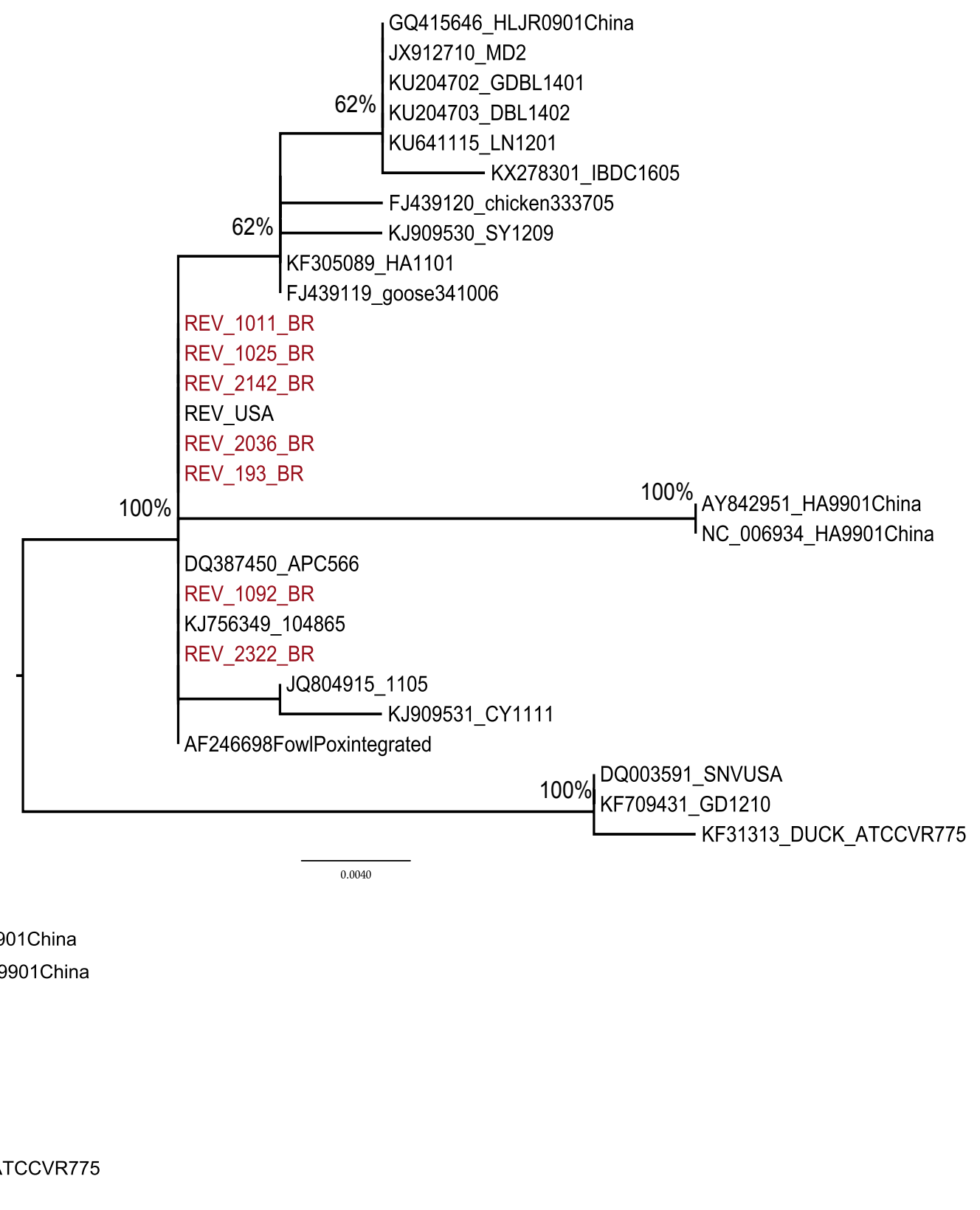

